\title{
Property (T) for groups acting on simplicial complexes through taking an "average" of Laplacian eigenvalues
}

\author{
Izhar Oppenheim
}

\begin{abstract}
This article generalizes the Żuk's criterion for property (T) and gives new criteria for property (T) (and for vanishing of higher $L^{2}$-cohomologies).
\end{abstract}

Mathematics Subject Classification (2010). Primary: 20F65; Secondary: 05C25.

Keywords. Property (T), cohomology, simplicial complex, Laplacian.

\section{Introduction}

1.1. Żuk's criterion and its generalizations toward proving property $(T)$. For a finite graph $L$ with a set of vertices $V_{L}$, the Laplacian of the graph $\Delta^{+}$is an operator on the space of real valued functions on $V_{L}$ which is defined as

$$
\Delta^{+} f(v)=f(v)-\frac{1}{m(v)} \sum_{u \sim v} f(u)
$$

where $m(v)$ is the valency of $v$ and $u \sim v$ means that there is an edge connecting $u$ and $v$. The Laplacian is a positive operator and we denote by $\lambda(L)$ its smallest positive eigenvalue. One can generalize the definition of the Laplacian so it will be defined for a simplicial complex $X$ of any dimension. For such a complex the Laplacian is again a positive operator and we denote by $\lambda(X)$ its smallest positive eigenvalue.

Ballmann and Światkowski in [2] and Żuk in [10] gave criteria for the vanishing of the $L^{2}$-cohomology of a group $\Gamma$ acting on a simplicial complex $X$, by considering the values of $\lambda$ for the links of $X$. More specifically in [2] and [10] the following theorems were proved: 
[2, Theorem 2.5]. Let $X$ be a locally finite simplicial complex of dimension $n$ and let $\Gamma$ be a group acting properly discontinuously by automorphisms on $X$. Assume that for every simplex $\eta$ of dimension $k-1$, the link of $\eta$, denoted by $X_{\eta}$, is a connected simplicial complex and that there is $\varepsilon>0$ such that

$$
\lambda\left(X_{\eta}\right) \geq \frac{k(n-k)}{k+1}+\varepsilon,
$$

then

$$
L^{2} H^{k}(X, \rho)=0
$$

for any unitary representation $\rho$ of $\Gamma$.

[10, Theorem 1]. Let $X$ be a locally finite simplicial complex of dimension 2 and let $\Gamma$ be a group acting properly discontinuously and freely by automorphisms on $X$. Assume that for every vertex $v$, the link of $v$, denoted by $X_{v}$, is a connected graph and that for every two connected vertices $u, v$ in $X$ we have the inequality

$$
\frac{\lambda\left(X_{u}\right)+\lambda\left(X_{v}\right)}{2}>\frac{1}{2},
$$

then

$$
L^{2} H^{1}(X, \rho)=0
$$

for any unitary representation $\rho$ of $\Gamma$.

In [4], the above theorems were generalized by Dymara and Januszkiewicz to a more general setting in which $\Gamma$ isn't necessarily discrete but just locally compact and unimodular.

If $\Gamma$ acts cocompactly and properly discontinuously by automorphisms on a contractible simplicial complex $X$, then the following are equivalent:

(1) $\Gamma$ has property $(\mathrm{T})$;

(2) $L^{2} H^{1}(X, \rho)=0$ for any unitary representation $\rho$ of $\Gamma$ (see [3]);

(3) $L^{2} \mathcal{H}^{1}(X, \rho)=0$ for any unitary representation $\rho$ of $\Gamma$, where $L^{2} \mathcal{H}^{1}(X, \rho)$ is the first reduced $L^{2}$-cohomology (see [3] and [8]).

Therefore the above theorems give criteria for property (T) when $\Gamma$ acts cocompactly and properly on a contractible locally finite simplicial complex of dimension 2. 
1.2. New criterion for property (T). In this article we shall generalize the above approach to get new criteria for vanishing of $L^{2}$-cohomologies for groups acting on simplicial complexes. The generalization is basically some sort of averaging along a simplex of the first positive eigenvalues of the Laplacian at the links. For the 2-dimensional case we get the following theorem:

Theorem 1. Let $\Gamma$ be a locally compact, unimodular group of automorphisms of $X$ acting cocompactly and properly on $X$ such that $X$ is a locally finite contractible 2-dimensional simplicial complex. For a 1-simplex $(u, v)$ of $X$ denote

$$
S_{(u, v)}=\lambda\left(X_{u}\right)+\lambda\left(X_{v}\right)-1 .
$$

If for every 2-simplex $(u, v, w)$ of $X$ the following holds:

- $S_{(u, v)}+S_{(v, w)}+S_{(u, w)}>0$,

- $S_{(u, v)} S_{(u, w)}+S_{(u, v)} S_{(v, w)}+S_{(u, w)} S_{(v, w)}>0$,

then $\Gamma$ has property $(\mathrm{T})$.

The difference between the our criterion stated above and Żuk's criterion is the extent of the eigenvalue averaging: Żuk's criterion averages the first Laplacian eigenvalue on edges, while the criterion stated above "averages" the first Laplacian eigenvalue on triangles. In the notation of the theorem above, Żuk's criterion can be stated as $S_{(u, v)}>0$ for each edge $(u, v)$ and therefore it is clear that Żuk's criterion is more restrictive than our criterion. Note that our criterion has an advantage only in cases where for each triangle $(u, v, w)$, there is an edge $(u, v)$ such that $S_{(u, v)}<0$. For instance in $\widetilde{A}_{2}$ buildings where $\lambda_{u}>\frac{1}{2}$ for every vertex $u$, our criterion has no advantage over Żuk's criterion.

There are some interesting examples for the above theorem coming from the exotic affine buildings. Here is one such example: in [6] it is shown that the Lyons group (which is a sporadic finite simple group) acts on a finite simplicial complex $Y$ and that the universal cover of $Y$ is an exotic affine building. This yields an extension of the Lyons group by $\pi_{1}(Y)$ :

$$
0 \longrightarrow \pi_{1}(Y) \longrightarrow \text { Lyons } \longrightarrow \text { Lyons } \longrightarrow 0 .
$$

As it turns out, Żuk's criterion doesn't show that $\pi_{1}(Y)$ has property (T) but our criterion holds and we are able to prove property $(\mathrm{T})$ for $\pi_{1}(Y)$ and Lyons. More examples regarding exotic affine buildings in and hyperbolic Kac-Moody groups where our criterion holds and Żuk's criterion fails are given in Section 4. 
Finally, let us remark that our results are stated in this introduction only for the vanishing of the first cohomology (property (T)), because it is easiest to present and is usually considered the most interesting case. In this article, we shall present a method of "averaging" Laplacian eigenvalue along simplices that gives a vanishing result for higher cohomologies. The most accessible case (in terms of finding the Laplacian eigenvalue and calculating the "average") is "averaging" the eigenvalue of 1-dimensional links over the top dimensional simplices to give raise to a vanishing of the $n-1$ cohomology (where $n$ is the top dimension).

Structure of the paper. Section 2 is devoted to introducing the framework developed in [2], Section 3 contains the vanishing results for $L^{2}$-cohomologies relaying on Laplacian eigenvalue and Section 4 contains examples of groups in which Theorem 1 proves property $(\mathrm{T})$.

Acknowledgements. The author wishes to thank his advisor Uri Bader, for many useful conversations and much encouragement along the way. The author would also like to thank the Technion - Israel Institute of Technology in which a large portion of this research was done as part of the author's Ph.D. thesis research.

\section{Framework}

Here we introduce the framework constructed in [2] and slightly generalized in [4] ([2] deals only with the case where the group acting is discrete, the generalization to the locally compact case was done in [4]). Throughout this section, $X$ is a locally finite simplicial complex of dimension $n$ such that all the links of $X$ are connected, excluding the 0 -dimensional ones. $\Gamma$ is a locally compact, properly discontinuous, unimodular group of automorphisms of $X$ such that the action of $\Gamma$ on $X$ is proper and $\rho$ is a unitary representation of $\Gamma$ on a complex Hilbert space $H$.

2.1. General settings. Following [2] we introduce the following notations.

(1) For $0 \leq k \leq n$, denote by $\Sigma(k)$ the set of ordered $k$-simplices (i.e. $\sigma \in \Sigma(k)$ is and ordered $k+1$-tuple of vertices) and choose a set $\Sigma(k, \Gamma) \subseteq \Sigma(k)$ of representatives of $\Gamma$-orbits. 
(2) For a simplex $\sigma \in \Sigma(k)$ denote by $m(\sigma)$ the number of $n$-simplices containing $\sigma$ without ordering, i.e. if $\sigma=\left(v_{0}, \ldots, v_{k}\right)$ then $m(\sigma)$ is the number of different unordered $n$-simplices which contain $\left\{v_{0}, \ldots, v_{k}\right\}$. We shall assume that $m(\sigma) \geq 1$ for every $\sigma$.

(3) For $\tau=\left(w_{0}, \ldots, w_{k}\right), \sigma=\left(v_{0}, \ldots, v_{l}\right)$ such that $k \leq l$, we denote $\tau \subset \sigma$ if $\left\{w_{0}, \ldots, w_{k}\right\} \subset\left\{v_{0}, \ldots, v_{l}\right\}$ and $\tau \sqsubset \sigma$ if there is a monotone function

$$
f:\{0, \ldots, k\} \longmapsto\{0, \ldots, l\}
$$

such that

$$
w_{i}=v_{f(i)} .
$$

(4) For a simplex $\sigma \in \Sigma(k)$, denote by $\Gamma_{\sigma}$ the stabilizer of $\sigma$ and by $\left|\Gamma_{\sigma}\right|$ the measure of $\Gamma_{\sigma}$ with respect to the Haar measure.

(5) For $0 \leq k \leq n$, denote by $C^{k}(X, \rho)$ the space of simplicial $k$-cochains of $X$ which are twisted by $\rho$, that is, $\phi \in C^{k}(X, \rho)$ is an alternating map on ordered $k$-simplices of $X$ with values in $H$ such that

$$
\rho(\gamma) \phi(x)=\phi(\gamma x), \quad \text { for all } \gamma \in \Gamma, x \in \Sigma(k) .
$$

For $\phi \in C^{k}(X, \rho)$ define $\phi$ to be square integrable $\bmod \Gamma$ if

$$
\|\phi\|^{2}:=\sum_{\sigma \in \Sigma(k, \Gamma)} \frac{m(\sigma)}{(k+1) !\left|\Gamma_{\sigma}\right|}\langle\phi(\sigma), \phi(\sigma)\rangle<\infty
$$

Denote by $L^{k}(X, \rho)$ the space of all square integrable cochains in $C^{k}(X, \rho)$. On $L^{k}(X, \rho)$ there is an Hermitian form given by

$$
\langle\phi, \psi\rangle:=\sum_{\sigma \in \Sigma(k, \Gamma)} \frac{m(\sigma)}{(k+1) !\left|\Gamma_{\sigma}\right|}\langle\phi(\sigma), \psi(\sigma)\rangle, \quad \phi, \psi \in L^{k}(X, \rho) .
$$

Note that if $\Gamma$ acts cocompactly on $X$ then $L^{k}(X, \rho)=C^{k}(X, \rho)$. To distinguish the norm of $L^{k}(X, \rho)$ from the norm of $H$ we will use |.| for the norm of $H$ (i.e. $\langle\phi(\sigma), \phi(\sigma)\rangle=|\phi(\sigma)|^{2}$ ).

For $0 \leq k<n$, the differential

$$
d: C^{k}(X, \rho) \longrightarrow C^{k+1}(X, \rho)
$$

is given by

$$
d \phi(\sigma):=\sum_{i=0}^{k+1}(-1)^{i} \phi\left(\sigma_{i}\right), \sigma \in \Sigma(k+1) .
$$

Where $\sigma_{i}=\left(v_{0}, \ldots, \hat{v}_{i}, \ldots, v_{k+1}\right)$ for $\left(v_{0}, \ldots, v_{k+1}\right)=\sigma \in \Sigma(k+1)$. 
Proposition 2.1. In [2] the following facts are proved.

(1) [2, Proposition 1.5] When the differential is restricted to

$$
d: L^{k}(X, \rho) \longrightarrow L^{k+1}(X, \rho)
$$

it is a bounded operator.

(2) [2, Proposition 1.6] The adjoint operator of $d$, denoted by

$$
\delta: L^{k+1}(X, \rho) \longrightarrow L^{k}(X, \rho)
$$

is

$$
\delta \phi(\tau)=\sum_{\substack{v \in \Sigma(0), v \tau \in \Sigma(k+1)}} \frac{m(v \tau)}{m(\tau)} \phi(v \tau), \quad \tau \in \Sigma(k),
$$

where $v \tau=\left(v, v_{0}, \ldots, v_{k}\right)$ for $\tau=\left(v_{0}, \ldots, v_{k}\right)$.

(3) [2, Corollary 1.7] For $\phi \in L^{k}(X, \rho)$ and $\sigma \in \Sigma(k)$,

$$
\delta d \phi(\sigma)=(n-k) \phi(\sigma)-\sum_{\substack{v \in \Sigma(0), v \sigma \in \Sigma(k+1)}} \sum_{0 \leq i \leq k}(-1)^{i} \frac{m(v \sigma)}{m(\sigma)} \phi\left(v \sigma_{i}\right) .
$$

Remark 2.2. Note that if $X$ is a 1-dimensional connected graph, $k=0$ and $H=\mathbb{R}$, then $\delta d$ is the (classical) graph Laplacian.

2.2. $L^{\mathbf{2}}$-cohomology. The $L^{2}$-cohomology of $X$ with respect to $\rho$ is defined as

$$
L^{2} H^{k}(X, \rho)=\operatorname{ker}\left(d \mid L^{k}(X, \rho)\right) / \operatorname{im}\left(d \mid L^{k-1}(X, \rho)\right)
$$

and the reduced $L^{2}$-cohomology of $X$ with respect to $\rho$ is defined as

$$
\operatorname{ker}\left(d \mid L^{k}(X, \rho)\right) / \overline{\operatorname{im}\left(d \mid L^{k-1}(X, \rho)\right)} .
$$

Recall that if $\Gamma$ acts cocompactly on a contractible $X$ then

$$
L^{2} H^{k}(X, \rho)=L^{2} H^{k}(\Gamma, \rho) .
$$

Let

$$
\Delta^{+}=\delta d, \Delta^{-}=d \delta, \Delta=\Delta^{+}+\Delta^{-} .
$$

Define the space of $L^{2} \mathcal{H}^{k}(X, \rho)$ of square integrable $\bmod \Gamma$ harmonic $k$-forms twisted by $\rho$ to be

$$
\begin{aligned}
L^{2} \mathcal{H}^{k}(X, \rho) & =\operatorname{ker}\left(\Delta \mid L^{k}(X, \rho)\right) \\
& =\operatorname{ker}\left(\Delta^{+} \mid L^{k}(X, \rho)\right) \cap \operatorname{ker}\left(\Delta^{-} \mid L^{k}(X, \rho)\right) .
\end{aligned}
$$


We have

$$
\begin{aligned}
& \left(\operatorname{ker}\left(\Delta^{+} \mid L^{k}(X, \rho)\right)\right)^{\perp}=\overline{\operatorname{im}\left(\Delta^{+} \mid L^{k}(X, \rho)\right)}=\overline{\operatorname{im}\left(\delta \mid L^{k+1}(X, \rho)\right)}, \\
& \left(\operatorname{ker}\left(\Delta^{-} \mid L^{k}(X, \rho)\right)\right)^{\perp}=\overline{\operatorname{im}\left(\Delta^{-} \mid L^{k}(X, \rho)\right)}=\overline{\operatorname{im}\left(d \mid L^{k-1}(X, \rho)\right)},
\end{aligned}
$$

and the orthogonal decompositions

$$
\begin{aligned}
& \operatorname{ker}\left(\Delta^{+} \mid L^{k}(X, \rho)\right)=L^{2} \mathcal{H}^{k}(X, \rho) \oplus \overline{\operatorname{im}\left(\Delta^{-} \mid L^{k}(X, \rho)\right)}, \\
& \operatorname{ker}\left(\Delta^{-} \mid L^{k}(X, \rho)\right)=L^{2} \mathcal{H}^{k}(X, \rho) \oplus \overline{\operatorname{im}\left(\Delta^{+} \mid L^{k}(X, \rho)\right)}
\end{aligned}
$$

In particular,

$$
L^{2} \mathcal{H}^{k}(X, \rho)=\operatorname{ker}\left(d \mid L^{k}(X, \rho)\right) / \overline{\operatorname{im}\left(d \mid L^{k-1}(X, \rho)\right)} .
$$

So $L^{2} \mathcal{H}^{k}(X, \rho)$ equals the reduced cohomology.

2.3. Localization. Let $\left(v_{0}, \ldots, v_{j}\right)=\tau \in \Sigma(j)$, denote by $X_{\tau}$ the link of $\tau$ in $X$, that is, the subcomplex of dimension $n-j-1$ consisting on simplices $\sigma=\left(w_{0}, \ldots, w_{k}\right)$ such that $\left\{v_{0}, \ldots, v_{j}\right\},\left\{w_{0}, \ldots, w_{k}\right\}$ are disjoint as sets and $\left(v_{0}, \ldots, v_{j}, w_{0}, \ldots, w_{k}\right)=\tau \sigma \in \Sigma(j+k+1)$. The isotropy group $\Gamma_{\tau}$ acts by automorphisms on $X_{\tau}$ and if we denote by $\rho_{\tau}$ the restriction of $\rho$ to $\Gamma_{\tau}$, we get that $\rho_{\tau}$ is a unitary representation of $\Gamma_{\tau}$. Note that since $X$ is locally finite and the action of $\Gamma$ on $X$ is proper, we get that $X_{\tau}$ is finite and $\Gamma_{\tau}$ is compact. Denote as in the general settings:

(1) for $0 \leq k \leq n-j-1$, denote by $\Sigma_{\tau}(k)$ the set of ordered $k$-simplices and choose a set $\Sigma_{\tau}\left(k, \Gamma_{\tau}\right) \subseteq \Sigma_{\tau}(k)$ of representatives of $\Gamma_{\tau}$-orbits;

(2) for a simplex $\sigma \in \Sigma_{\tau}(k)$ denote by $m_{\tau}(\sigma)$ the number of $n-j-1$-simplices containing $\sigma$ in $X_{\tau}$. Note that $m_{\tau}(\sigma)=m(\tau \sigma)$ and by our previous assumption, $m_{\tau}(\sigma) \geq 1$ for every $\sigma$;

(3) for a simplex $\sigma \in \Sigma_{\tau}(k)$, denote by $\Gamma_{\tau \sigma}$ the stabilizer of $\sigma$ in $\Gamma_{\tau}$;

(4) for $0 \leq k \leq n-j-1$, denote by $C^{k}\left(X_{\tau}, \rho_{\tau}\right)$ the space of simplicial $k$-cochains of $X_{\tau}$ which are twisted by $\rho_{\tau}$.

We also define $L^{k}\left(X_{\tau}, \rho_{\tau}\right), d_{\tau}, \delta_{\tau}, \Delta_{\tau}^{+}, \Delta_{\tau}^{-}, \Delta_{\tau}$ and the $L^{2}$-cohomology as before. Note that since $X_{\tau}$ is finite we get that $L^{k}\left(X_{\tau}, \rho_{\tau}\right)=C^{k}\left(X_{\tau}, \rho_{\tau}\right)$.

Define the localization map

$$
C^{k}(X, \rho) \longrightarrow C^{k-j-1}\left(X_{\tau}, \rho_{\tau}\right), \quad \phi \longmapsto \phi_{\tau},
$$

where $\phi_{\tau}(\sigma)=\phi(\tau \sigma)$. 


\subsection{Further results from [2], [4]}

Proposition 2.3. In [2],[4] following results were proven.

(1) [2, Lemma 1.3], [4, Lemma 3.3] For $0 \leq l<k \leq n$, let $f=f(\tau, \sigma)$ be a $\Gamma$-invariant function on the set of pairs $(\tau, \sigma)$, where $\tau$ is an ordered $l$-simplex and $\sigma$ is an ordered $k$-simplex with $\tau \subset \sigma$ Then

$$
\sum_{\sigma \in \Sigma(k, \Gamma)} \sum_{\substack{\tau \in \Sigma(l) \\ \tau \subset \sigma}} \frac{f(\tau, \sigma)}{\left|\Gamma_{\sigma}\right|}=\sum_{\tau \in \Sigma(l, \Gamma)} \sum_{\substack{\sigma \in \Sigma(k) \\ \tau \subset \sigma}} \frac{f(\tau, \sigma)}{\left|\Gamma_{\tau}\right|} .
$$

(2) [2, equality (1.9)] For $\psi \in C^{l}\left(X_{\tau}, \rho_{\tau}\right)$ we have

$$
\begin{aligned}
\|\psi\|^{2} & =\sum_{\eta \in \Sigma_{\tau}\left(l, \Gamma_{\tau}\right)} \frac{m_{\tau}(\eta)}{\left|\Gamma_{\tau \eta}\right|(l+1) !}|\psi(\eta)|^{2} \\
& =\frac{1}{(l+1) !\left|\Gamma_{\tau}\right|} \sum_{\eta \in \Sigma_{\tau}(l)} m(\tau \eta)|\psi(\eta)|^{2} .
\end{aligned}
$$

(3) [2, Lemma 1.11] For $0 \leq k \leq n, \tau \in \Sigma(k-1)$ and $\phi \in L^{k}(X, \rho)$, denote by $\phi_{\tau}^{0}$ the component of $\phi_{\tau}$ in the subspace of constant maps in $C^{0}\left(X_{\tau}, \rho_{\tau}\right)$ then

$$
\left\|\phi_{\tau}^{0}\right\|^{2}=\frac{m(\tau)}{(n-k+1)\left|\Gamma_{\tau}\right|}|\delta \phi(\tau)|^{2}
$$

and therefore

$$
\|\delta \phi\|^{2}=\sum_{\tau \in \Sigma(k-1, \Gamma)} \frac{n-k+1}{k !}\left\|\phi_{\tau}^{0}\right\|^{2} .
$$

(4) [2, Theorem 1.12] Let $0 \leq j<k<n$ and $\phi \in L^{k}(X, \rho)$ then

$$
k !\left(\|d \phi\|^{2}-(n-k)\|\phi\|^{2}\right)=(k-j-1) ! \sum_{\tau \in \Sigma(j, \Gamma)}\left\|d_{\tau} \phi_{\tau}\right\|^{2}-(n-k)\left\|\phi_{\tau}\right\|^{2} .
$$

[2, Corollary 1.13] For $0<k<n$ and $\tau \in \Sigma(k-1)$ define a quadratic form $Q_{\tau}$ on $C^{0}\left(X_{\tau}, \rho_{\tau}\right)$ by

$$
\begin{aligned}
Q_{\tau}(\psi) & =\left\|d_{\tau} \psi\right\|^{2}-\frac{k}{k+1}(n-k)\|\psi\|^{2} \\
& =\left\langle\Delta_{\tau}^{+} \psi, \psi\right\rangle-\frac{k}{k+1}(n-k)\|\psi\|^{2} .
\end{aligned}
$$

Then for every $\phi \in L^{k}(X, \rho)$ we have

$$
k !\|d \phi\|^{2}=\sum_{\tau \in \Sigma(k-1, \Gamma)} Q_{\tau}\left(\phi_{\tau}\right) .
$$


2.5. Vanishing of $\boldsymbol{L}^{\mathbf{2}}$-cohomologies. The idea (taken from [2]) for proving that $L^{2} H^{k}(X, \rho)=0$ for every $\rho$ goes as follows: prove that there is an $\varepsilon>0$ such that

$$
\|d \phi\|^{2}=0 \Longrightarrow\|\delta \phi\|^{2} \geq \varepsilon\|\phi\|^{2}, \quad \text { for all } \phi \in L^{k}(X, \rho) .
$$

This will imply that $\operatorname{ker}\left(d \mid L^{k}(X, \rho)\right) \cap \operatorname{ker}\left(\delta \mid L^{k}(X, \rho)\right)=0$ and therefore $\operatorname{ker}\left(\Delta^{+} \mid L^{k}(X, \rho)\right) \cap \operatorname{ker}\left(\Delta^{-} \mid L^{k}(X, \rho)\right)=0$ so we get $L^{2} \mathcal{H}^{k}(X, \rho)=0$. Also, by the above inequality, we get that the image of $\Delta^{-} \mid L^{k}(X, \rho)$ is closed in $\operatorname{ker}\left(\Delta^{+} \mid L^{k}(X, \rho)\right)$. Hence $\operatorname{ker}\left(\Delta^{+} \mid L^{k}(X, \rho)\right)=\operatorname{im}\left(\Delta^{-} \mid L^{k}(X, \rho)\right)$ and therefore $L^{2} H^{k}(X, \rho)=0$. In particular, one could prove the vanishing of $L^{k}(X, \rho)$ for every $\rho$ by showing that there is an $\varepsilon>0$ such that for every representation $\rho$ the following holds:

$$
\|d \phi\|^{2}+\|\delta \phi\|^{2} \geq \varepsilon\|\phi\|^{2}, \quad \text { for all } \phi \in L^{k}(X, \rho) .
$$

In the same manner, to prove that $L^{2} \mathcal{H}^{k}(X, \rho)=0$ for every $\rho$, it is enough to prove

$$
\|\delta \phi\|^{2}=0 \Longrightarrow\|d \phi\|^{2}>0, \quad \text { for all } \phi \in L^{k}(X, \rho) .
$$

\section{Taking an "average" of Laplacian eigenvalues}

In this section we shall find criteria for the vanishing of $L^{2}$-cohomologies using the eigenvalues for the Laplacians.

Definition 3.1. For $0 \leq k<l$ integers define $M_{k}^{l}$ as following: let $\gamma$ be an ordered simplex of dimension $l$ denote $F=\{\sigma \sqsubset \gamma: \operatorname{dim}(\sigma)=k\}, F^{\prime}=$ $\{v \sqsubset \gamma: \operatorname{dim}(v)=k+1\}$. Denote by $[v: \sigma]$ the sign of $\sigma$ in $v$, i.e. if $v=$ $\left(v_{0}, \ldots, v_{k+1}\right), \sigma=\left(v_{0}, \ldots, \hat{v}_{i}, \ldots, v_{k+1}\right)$, then $[v: \sigma]=(-1)^{i} . M_{k}^{l}$ is a matrix indexed by $F \cup F^{\prime}$ defined as

$$
\left(M_{k}^{l}\right)_{\alpha, \beta}= \begin{cases}x_{\alpha} & \text { if } \alpha=\beta \in F, \\ {[\beta: \alpha]} & \text { if } \alpha \sqsubset \beta \text { when } \alpha \in F \text { and } \beta \in F^{\prime}, \\ {[\alpha: \beta]} & \text { if } \beta \sqsubset \alpha \text { when } \beta \in F \text { and } \alpha \in F^{\prime}, \\ 0 & \text { otherwise. }\end{cases}
$$

Define $p_{k}^{l}\left(x_{\sigma}\right)$ to be the multi-polynomial with variables indexed by $F$ as

$$
p_{k}^{l}\left(x_{\sigma}\right)=\operatorname{det}\left(M_{k}^{l}\right)
$$


Example 3.2. For $l=k+1$,

$$
p_{k}^{l}\left(x_{\sigma}\right)=-\sum_{\sigma \in F} \prod_{\sigma^{\prime} \in F, \sigma^{\prime} \neq \sigma} x_{\sigma^{\prime}}
$$

notice that in this case we get

$$
p_{k}^{l}\left(x_{\sigma}\right)=-\nabla \cdot\left(\prod_{\sigma^{\prime} \in F} x_{\sigma^{\prime}}\right)
$$

Remark 3.3. Notice that if $2(k+1)<l+1$ then

$$
|F|=\left(\begin{array}{l}
l+1 \\
k+1
\end{array}\right) \leq\left(\begin{array}{l}
l+1 \\
k+2
\end{array}\right)=\left|F^{\prime}\right|
$$

and therefore $p_{k}^{l}$ is constant (if $2(k+1)<l$ then $p_{k}^{l} \equiv 0$ ).

For every simplex $\tau$ of dimension $k-1$ denote by $\lambda_{\tau}$ the smallest positive eigenvalue of $\Delta_{\tau}^{+}$on $X_{\tau}$ (the link of $\tau$ ) also denote

$$
\overline{\lambda_{\tau}}=\lambda_{\tau}-\frac{k(n-k)}{k+1}
$$

Now for a simplex $\sigma$ of dimension $k$ denote

$$
S_{\sigma}=\sum_{\substack{\tau \in \Sigma(k-1), \tau \sqsubset \sigma}} \overline{\lambda_{\tau}}
$$

Theorem 3.4. If there is an $l>k$ and $\varepsilon>0$ such that for any $\gamma \in \Sigma(l, \Gamma)$ one has

$$
p_{k}^{l}\left(\lambda-S_{\sigma}\right)=0 \Longrightarrow \lambda \geq \varepsilon
$$

where $p_{k}^{l}\left(\lambda-S_{\sigma}\right)$ is the polynomial obtained by placing $x_{\sigma}=\lambda-S_{\sigma}$ for each $\sigma \subset \gamma$, then $L^{2} H^{k}(X, \rho)=0$ for every $\rho$.

Proof. We shall start with repeating the proof in [2].

Let $\phi \in L^{k}(X, \rho)$ then by Proposition 2.3 (4), one has

$$
k !\|d \phi\|^{2}=\sum_{\tau \in \Sigma(k-1, \Gamma)}\left\langle\Delta_{\tau}^{+}\left(\phi_{\tau}\right), \phi_{\tau}\right\rangle-\frac{k}{k+1}(n-k)\left\|\phi_{\tau}\right\|^{2} .
$$


Now denote as before $\phi_{\tau}^{0}$ to be the projection of $\phi_{\tau}$ on the space of constant function on $X_{\tau}$ and by $\phi_{\tau}^{1}$ its orthogonal complement. Note that since $\operatorname{ker}\left(\Delta_{\tau}^{+}\right)$is that space of constant function on $X_{\tau}$ (since $X_{\tau}$ is connected), we get that

$$
\left\langle\Delta_{\tau}^{+}\left(\phi_{\tau}\right), \phi_{\tau}\right\rangle=\left\langle\Delta_{\tau}^{+}\left(\phi_{\tau}^{1}\right), \phi_{\tau}^{1}\right\rangle \geq \lambda_{\tau}\left\|\phi_{\tau}^{1}\right\|^{2}
$$

Therefore

$$
\begin{aligned}
k !\|d \phi\|^{2} & \geq \sum_{\tau \in \Sigma(k-1, \Gamma)} \lambda_{\tau}\left\|\phi_{\tau}^{1}\right\|^{2}-\frac{k}{k+1}(n-k)\left\|\phi_{\tau}\right\|^{2} \\
& =\sum_{\tau \in \Sigma(k-1, \Gamma)} \lambda_{\tau}\left\|\phi_{\tau}\right\|^{2}-\lambda_{\tau}\left\|\phi_{\tau}^{0}\right\|^{2}-\frac{k}{k+1}(n-k)\left\|\phi_{\tau}\right\|^{2} .
\end{aligned}
$$

So we get

$$
k !\|d \phi\|^{2}+\sum_{\tau \in \Sigma(k-1, \Gamma)} \lambda_{\tau}\left\|\phi_{\tau}^{0}\right\|^{2} \geq \sum_{\tau \in \Sigma(k-1, \Gamma)} \overline{\lambda_{\tau}}\left\|\phi_{\tau}\right\|^{2}
$$

Note that since $\left\|d_{\tau}\right\|^{2} \leq 2(n-k)$ for every $\tau$ (see [2, Proposition 1.5]) then $\lambda_{\tau} \leq 2(n-k)$ for every $\tau$ and therefore

$$
\begin{aligned}
k ! \frac{2(n-k)}{n-k+1}\|\delta \phi\|^{2} & =\sum_{\tau \in \Sigma(k-1, \Gamma)} 2(n-k)\left\|\phi_{\tau}^{0}\right\|^{2} \\
& \geq \sum_{\tau \in \Sigma(k-1, \Gamma)} \lambda_{\tau}\left\|\phi_{\tau}^{0}\right\|^{2} .
\end{aligned}
$$

So we get

$$
k !\|d \phi\|^{2}+k ! \frac{2(n-k)}{n-k+1}\|\delta \phi\|^{2} \geq \sum_{\tau \in \Sigma(k-1, \Gamma)} \overline{\lambda_{\tau}}\left\|\phi_{\tau}\right\|^{2}
$$

Remark 3.5. At this stage, if we wanted to prove the result in [2] (every $\overline{\lambda_{\tau}} \geq \varepsilon$ implies the vanishing of the cohomology) we would be done, because then

$$
k !\|d \phi\|^{2}+k ! \frac{2(n-k)}{n-k+1}\|\delta \phi\|^{2} \geq \varepsilon(k+1) !\|\phi\|^{2} .
$$


Now we shall assume that $\phi \in \operatorname{ker}(d)$ and show that under the condition stated in the theorem we get that

$$
\frac{2(n-k)}{n-k+1}\|\delta \phi\|^{2} \geq \varepsilon\|\phi\|^{2}
$$

and that will finish the proof (using the idea stated in Section 2.5).

By definition and Proposition 2.3 (2) we get that

$$
\begin{aligned}
& \sum_{\tau \in \Sigma(k-1, \Gamma)} \overline{\lambda_{\tau}}\left\|\phi_{\tau}\right\|^{2}=\sum_{\tau \in \Sigma(k-1, \Gamma)} \overline{\lambda_{\tau}} \frac{1}{\left|\Gamma_{\tau}\right|} \sum_{\eta \in \Sigma_{\tau}(0)} m(\tau \eta)|\phi(\tau \eta)|^{2} \\
& =\sum_{\tau \in \Sigma(k-1, \Gamma)} \overline{\lambda_{\tau}} \frac{1}{\left|\Gamma_{\tau}\right|} \sum_{\substack{\sigma \in \Sigma(k), \tau \subset \sigma}} \frac{1}{(k+1) !} m(\sigma)|\phi(\sigma)|^{2}
\end{aligned}
$$

by Proposition 2.3 (1) we can change the order of summation and get

$$
\sum_{\sigma \in \Sigma(k, \Gamma)} \frac{m(\sigma)|\phi(\sigma)|^{2}}{(k+1) !\left|\Gamma_{\sigma}\right|} \sum_{\substack{\tau \in \Sigma(k-1), \tau \subset \sigma}} \overline{\lambda_{\tau}}=k ! \sum_{\sigma \in \Sigma(k, \Gamma)} \frac{m(\sigma)|\phi(\sigma)|^{2}}{(k+1) !\left|\Gamma_{\sigma}\right|} S_{\sigma} .
$$

Now note that

$$
(l+1) !\left(\begin{array}{c}
n-k \\
l-k
\end{array}\right) m(\sigma)=\sum_{\substack{\gamma \in \Sigma(l) \\
\sigma \subset \gamma}} m(\gamma)
$$

So we get

$$
\begin{aligned}
& k ! \sum_{\sigma \in \Sigma(k, \Gamma)} \frac{m(\sigma)|\phi(\sigma)|^{2}}{(k+1) !\left|\Gamma_{\sigma}\right|} S_{\sigma} \\
& =k ! \sum_{\sigma \in \Sigma(k, \Gamma)} \frac{|\phi(\sigma)|^{2}}{(k+1) !\left|\Gamma_{\sigma}\right|} S_{\sigma} \sum_{\substack{\gamma \in \Sigma(l), \sigma \subset \gamma}} \frac{m(\gamma)}{(l+1) !\left(\begin{array}{l}
n-k \\
l-k
\end{array}\right)} .
\end{aligned}
$$

and by changing the order of summation again we get

$$
\frac{k !}{(k+1) !} \sum_{\gamma \in \Sigma(l, \Gamma)} \frac{m(\gamma)}{(l+1) !\left(\begin{array}{c}
n-k \\
l-k
\end{array}\right)\left|\Gamma_{\gamma}\right|} \sum_{\substack{\sigma \in \Sigma(k), \sigma \subset \gamma}} S_{\sigma}|\phi(\sigma)|^{2} .
$$


Now we shall show that, under the conditions of the theorem, for every $\gamma \in \Sigma(l, \Gamma)$ we have the the following inequality

$$
\sum_{\sigma \in \Sigma(k), \sigma \subset \gamma} S_{\sigma}|\phi(\sigma)|^{2} \geq \varepsilon \sum_{\substack{\sigma \in \Sigma(k), \sigma \subset \gamma}}|\phi(\sigma)|^{2}
$$

and that will complete the proof because then we have

$$
\begin{aligned}
& \frac{k !}{(k+1) !} \sum_{\gamma \in \Sigma(l, \Gamma)} \frac{m(\gamma)}{(l+1) !\left(\begin{array}{c}
n-k \\
l-k
\end{array}\right)\left|\Gamma_{\gamma}\right|} \sum_{\substack{\sigma \in \Sigma(k), \sigma \subset \gamma}} S_{\sigma}|\phi(\sigma)|^{2} \\
& \geq \frac{k !}{(k+1) !} \sum_{\gamma \in \Sigma(l, \Gamma)} \frac{m(\gamma)}{(l+1) !\left(\begin{array}{c}
n-k \\
l-k
\end{array}\right)\left|\Gamma_{\gamma}\right|} \sum_{\substack{\sigma \in \Sigma(k), \sigma \subset \gamma}} \varepsilon|\phi(\sigma)|^{2} \\
& \varepsilon \frac{k !}{(k+1) !} \sum_{\sigma \in \Sigma(k, \Gamma)} \frac{|\phi(\sigma)|^{2}}{\left|\Gamma_{\sigma}\right|} \sum_{\substack{\gamma \in \Sigma(l), \sigma \subset \gamma}} \frac{m(\gamma)}{(l+1) !\left(\begin{array}{c}
n-k \\
l-k
\end{array}\right)} \\
& \varepsilon k ! \sum_{\sigma \in \Sigma(k, \Gamma)} \frac{m(\sigma)|\phi(\sigma)|^{2}}{(k+1) !\left|\Gamma_{\sigma}\right|}=\varepsilon k !\|\phi\|^{2},
\end{aligned}
$$

and therefore we get

$$
\frac{2(n-k)}{n-k+1}\|\delta \phi\|^{2} \geq \varepsilon\|\phi\|^{2} .
$$

So we are left with proving the following inequality - for every $\phi \in L^{k}(X, \rho) \cap$ $\operatorname{ker}(d)$ and for every $\gamma \in \Sigma(l, \Gamma)$ one has (under the conditions of the theorem):

$$
\sum_{\sigma \in \Sigma(k), \sigma \subset \gamma} S_{\sigma}|\phi(\sigma)|^{2} \geq \varepsilon \sum_{\sigma \in \Sigma(k), \sigma \subset \gamma}|\phi(\sigma)|^{2} .
$$

Fix $\gamma \in \Sigma(l, \Gamma)$, first note that since $\phi$ is alternating we get that

$$
\sum_{\sigma \in \Sigma(k), \sigma \subset \gamma} S_{\sigma}|\phi(\sigma)|^{2}=(k+1) ! \sum_{\sigma \in \Sigma(k), \sigma \sqsubset \gamma} S_{\sigma}|\phi(\sigma)|^{2}
$$

and

$$
\sum_{\sigma \in \Sigma(k), \sigma \subset \gamma}|\phi(\sigma)|^{2}=(k+1) ! \sum_{\sigma \in \Sigma(k), \sigma \sqsubset \gamma}|\phi(\sigma)|^{2}
$$

therefore it is enough to prove that

$$
\sum_{\sigma \in \Sigma(k), \sigma \sqsubset \gamma} S_{\sigma}|\phi(\sigma)|^{2} \geq \varepsilon \sum_{\sigma \in \Sigma(k), \sigma \sqsubset \gamma}|\phi(\sigma)|^{2} .
$$


Now we shall need the following simple but useful lemma, which is a straightforward generalization of [2, Lemma 2.3].

Lemma 3.6. For a finite set $F=\left\{x_{1}, \ldots, x_{n}\right\}$, denote by $C(F, H)$ the space of maps from $F$ to $H$. On that space there is a natural inner product:

$$
\langle\phi, \psi\rangle=\sum_{k=1}^{n}\left\langle\phi\left(x_{k}\right), \psi\left(x_{k}\right)\right\rangle
$$

and denote by $\|$.$\| the norm induced by this inner product. Note that C(F, H)=$ $C(F, \mathbb{R}) \otimes H$ (as a Hilbert space). Let $T_{j}, j=1, \ldots, m$ and $M_{i}, i=1, \ldots, l$ be bounded linear operators on $C(F, H)$ so there are operators $T_{j, \mathbb{R}}, M_{i, \mathbb{R}}$ on $C(F, \mathbb{R})$ such that $T_{j}=T_{j, \mathbb{R}} \otimes i d, M_{i}=M_{i, \mathbb{R}} \otimes i d$. Let $a_{1}, \ldots, a_{j} \in \mathbb{R}$ be constants, then if there is an $\varepsilon$ such that

$$
\phi \in \cap \operatorname{ker}\left(M_{i, \mathbb{R}}\right) \Longrightarrow \sum_{j} a_{j}\left\|T_{j, \mathbb{R}} \phi\right\|^{2} \geq \varepsilon\|\phi\|^{2}, \quad \text { for all } \phi \in C(F, \mathbb{R}) .
$$

Then also

$$
\phi \in \cap \operatorname{ker}\left(M_{i}\right) \Longrightarrow \sum_{j} a_{j}\left\|T_{j} \phi\right\|^{2} \geq \varepsilon\|\phi\|^{2}, \quad \text { for all } \phi \in C(F, H) .
$$

Proof. Choose $\left\{v_{\alpha}\right\}$ orthonormal basis of $H$. For every $\phi \in C(F, H)$ one has $\phi_{\alpha} \in C(X, \mathbb{R})$ such that $\phi=\sum \phi_{\alpha} \otimes v_{\alpha}$. Now note that $\phi \in \cap \operatorname{ker}\left(M_{i}\right)$ if and only if, for all $\alpha, \phi_{\alpha} \in \cap \operatorname{ker}\left(M_{i, \mathbb{R}}\right)$, so we get

$$
\begin{aligned}
\sum_{j} a_{j}\left\|T_{j} \phi\right\|^{2} & =\left\|\sum_{j} T_{j} \sum_{\alpha} \phi_{\alpha} \otimes v_{\alpha}\right\|^{2} \\
& =\sum_{\alpha} \sum_{j} a_{j}\left\|T_{j, \mathrm{R}} \phi_{\alpha}\right\|^{2} \\
& \geq \sum_{\alpha} \varepsilon\left\|\phi_{\alpha}\right\|^{2} \\
& =\varepsilon\|\phi\|^{2}, \quad \phi \in \cap \operatorname{ker}\left(M_{i}\right) .
\end{aligned}
$$

Now we can use the above lemma to reduce our problem to a much simpler one. Denote

$$
F=\{\sigma \in \Sigma(k): \sigma \sqsubset \gamma\}
$$

and

$$
F^{\prime}=\{v \in \Sigma(k+1): v \sqsubset \gamma\}
$$


and for $\phi \in L^{k}(X, \rho) \cap \operatorname{ker}(d)$ we can look at the restriction of $\phi$ to $F$. Note that $\phi \in \operatorname{ker}(d)$ implies that for every $v \in \Sigma(k+1)$ we have $d \phi(v)=0$ and in particular, for all $v \in F^{\prime}, d \phi(v)=0$. Therefore for every $v \in F^{\prime}$, we define $M_{v}$ acting on $C(F, H)$ as $M_{\nu} \phi=\sum(-1)^{i} \phi\left(v_{i}\right)$. For every $\sigma \in F$ define $T_{\sigma}$ acting on $C(F, H)$ as the projection on the space spanned by the indicator function of $\sigma$. If we can prove that for every $\phi \in C(F, H)$ the following holds

$$
\phi \in \cap \operatorname{ker}\left(M_{\nu}\right) \Longrightarrow \sum_{\sigma \sqsubset \gamma} S_{\sigma}\left\|T_{\sigma} \phi\right\|^{2} \geq \varepsilon\|\phi\|^{2},
$$

then in particular, for every $\phi \in C^{k}(X, \rho)$ we have

$$
\sum_{\sigma \in \Sigma(k), \sigma \sqsubset \gamma} S_{\sigma}|\phi(\sigma)|^{2} \geq \varepsilon \sum_{\sigma \in \Sigma(k), \sigma \sqsubset \gamma}|\phi(\sigma)|^{2} .
$$

By the above lemma, it is enough to prove that for $\phi \in C(F, \mathbb{R})$ we have

$$
\phi \in \cap \operatorname{ker}\left(M_{\nu}\right) \Longrightarrow \sum_{\sigma \sqsubset \gamma} S_{\sigma}\left\|T_{\sigma} \phi\right\|^{2} \geq \varepsilon\|\phi\|^{2}
$$

and if we denote $\phi(\sigma)=x_{\sigma}$ we get the following problem: prove that

$$
\sum_{\sigma \in \Sigma(k), \sigma \sqsubset \gamma} S_{\sigma} x_{\sigma}^{2} \geq \varepsilon \sum_{\sigma \in \Sigma(k), \sigma \sqsubset \gamma} x_{\sigma}^{2}
$$

under the constraints

$$
\sum_{i=0}^{k+1}(-1)^{i} x_{v_{i}}=0, \quad \text { for all } v \in F^{\prime} .
$$

since both sides of the inequality we are trying to prove are quadratic, without loss of generality it is enough to prove that

$$
\sum_{\sigma \in \Sigma(k), \sigma \sqsubset \gamma} S_{\sigma} x_{\sigma}^{2} \geq \varepsilon
$$

under the constraints

$$
\begin{aligned}
\sum_{\sigma \in \Sigma(k), \sigma \sqsubset \gamma} x_{\sigma}^{2} & =1, \\
\sum_{\sigma \in F, \sigma \sqsubset \nu}[v: \sigma] x_{\sigma} & =0, \quad \text { for all } v \in F^{\prime} .
\end{aligned}
$$


This is a problem of finding a minimum of a function in $\mathbb{R}^{|F|}$ under constraints which define a compact set in $\mathbb{R}^{|F|}$ and so we can use the Lagrange multiplier theorem. Define the Lagrange function to be

$$
\begin{aligned}
& \Lambda\left(x_{\sigma}, \mu_{\nu}, \lambda\right) \\
& \quad=\sum_{\sigma \in \Sigma(k), \sigma \sqsubset \gamma} S_{\sigma} x_{\sigma}^{2}-2 \sum_{\nu \in F^{\prime}} \mu_{\nu}\left(\sum_{\sigma \in F, \sigma \sqsubset v}[v: \sigma] x_{\sigma}-\lambda \sum_{\sigma \in \Sigma(k), \sigma \sqsubset \gamma} x_{\sigma}^{2}-1\right)
\end{aligned}
$$

(the 2 multiplying $\sum_{v \in F}$, is added for convenience)

So for every $\sigma \in F$ we get a equation by derivation of $\Lambda$ by $x_{\sigma}$ :

$$
2 S_{\sigma} x_{\sigma}-2 \lambda x_{\sigma}-2 \sum_{v \in F^{\prime}, \sigma \sqsubset v}[v: \sigma] \mu_{v}=0
$$

if we multiply every such equation by $x_{\sigma}$ and add them up (over all $\sigma \in F$ ) we get

$$
2 \sum_{\sigma \in \Sigma(k), \sigma \sqsubset \gamma} S_{\sigma} x_{\sigma}^{2}-2 \lambda\left(\sum_{\sigma \in \Sigma(k), \sigma \sqsubset \gamma} x_{\sigma}^{2}\right)-2 \sum_{\nu \in F^{\prime}} \mu_{\nu} \sum_{\sigma \in F, \sigma \sqsubset \nu}[v: \sigma] x_{\sigma}=0
$$

and by the equations coming from the constraints we get

$$
\sum_{\sigma \in \Sigma(k), \sigma \sqsubset \gamma} S_{\sigma} x_{\sigma}^{2}=\lambda
$$

So the minimum is some $\lambda$ which is a part of a vector $\left(x_{\sigma}, \mu_{v}, \lambda\right)$ which solves $\nabla \Lambda=0$.

Treat $\lambda$ as a parameter and consider the system of linear equations in $\left(x_{\sigma}, \mu_{v}\right)$

$$
\begin{gathered}
\left(\lambda-S_{\sigma}\right) x_{\sigma}+\sum_{\nu \in F^{\prime}, \sigma \sqsubset v}[v: \sigma] \mu_{v}=0, \\
\sum_{\sigma \in F, \sigma \sqsubset v}[v: \sigma] x_{\sigma}=0 .
\end{gathered}
$$

Note that from

$$
\sum_{\sigma \in \Sigma(k), \sigma \sqsubset \gamma} x_{\sigma}^{2}=1
$$

the minimum is obtained only if this system of equations has a non trivial solution, that is only if the determinant is zero, but this determinant is exactly $p_{k}^{l}\left(\lambda-S_{\sigma}\right)$ and by the conditions of the theorem we know that for every root $\lambda$ of this polynomial we have $\lambda \geq \varepsilon$ and so we are done. 
Now Theorem 1 is proven as a corollary:

Corollary 3.7. Let $\Gamma$ be a locally compact, properly discontinuous, unimodular group of automorphisms of $X$ acting cocompactly on $X$ such that $X$ is a locally finite contractible 2-dimensional simplicial complex. If for every $(u, v, w) \in$ $\Sigma(2, \Gamma)$ the following holds:

- $S_{(u, v)}+S_{(v, w)}+S_{(u, w)}>0$,

- $S_{(u, v)} S_{(u, w)}+S_{(u, v)} S_{(v, w)}+S_{(u, w)} S_{(v, w)}>0$,

then $\Gamma$ has property $(\mathrm{T})$.

Proof. By the above theorem it is enough to prove that for every $(u, v, w) \in$ $\Sigma(2, \Gamma)$ all the roots of $p_{1}^{2}\left(\lambda-S_{(u, v)}, \lambda-S_{(v, w)}, \lambda-S_{(u, w)}\right)$ are positive. We have

$$
\begin{aligned}
p_{1}^{2}(\lambda- & \left.S_{(u, v)}, \lambda-S_{(v, w)}, \lambda-S_{(u, w)}\right) \\
=(\lambda- & \left.S_{(u, v)}\right)\left(\lambda-S_{(v, w)}\right) \\
+ & \left(\lambda-S_{(u, v)}\right)\left(\lambda-S_{(u, w)}\right) \\
& +\left(\lambda-S_{(v, w)}\right)\left(\lambda-S_{(u, w)}\right)
\end{aligned}
$$

one can find the roots of the polynomial explicitly and the roots of this polynomial are positive if and only if

- $S_{(u, v)}+S_{(v, w)}+S_{(u, w)}>0$,

- $S_{(u, v)} S_{(u, w)}+S_{(u, v)} S_{(v, w)}+S_{(u, w)} S_{(v, w)}>0$.

Remark 3.8. The above corollary gives some intuition to the nature of the criteria given in theorem 3.4. Intuitively speaking, the condition takes an "average" of the eigenvalues of Laplacians corresponding to different links of a simplex and if this "average" is large enough for each simplex, then the cohomology vanishes.

\section{Examples}

In this section we'll present examples for which our 2 dimensional criterion for property (T) holds and the Żuk's criterion fails. Both type of examples will involve Tits buildings. We shall not present the theory of Tits buildings apart from saying that they are simplicial complexes for which that the links of dimension 1 are generalized polygons introduced below. The interested reader can find a good introduction to the subject of buildings in [1]. 
4.1. Generalized polygons. A generalized $m$-gon (or in another name, a 1-dimensional spherical building) is a connected bipartite graph $L=(V, E)$ of diameter $m$ and girth $2 m$ in which each vertex lies on at least two edges. Denote $V=V_{1} \cup V_{2}$ were $V_{1}, V_{2}$ are the two sides of the graph (there is an edge between two vertices only if one belong to $V_{1}$ and the other to $V_{2}$ ). A generalized $m$-gon is said to have parameters $(s, t)$ if every vertex in $V_{1}$ has valency $s+1$ and every vertex in $V_{2}$ has valency $t+1$. A generalized $m$-gon is called thick if $s \geq 2, t \geq 2$. A theorem by Feit and Higman [5] states that a thick $m$-gon exists only if $m=2,3,4,6,8$. Moreover, Feit and Higman computed the smallest positive eigenvalue for the Laplacian on general $m$-gon of type $(s, t)$ and those are given in the list below.

(1) For $m=2$ the generalized $m$-gon is a complete bipartite graph and the smallest positive eigenvalue of the Laplacian is always 1 (is does not depend on $(s, t))$.

(2) For $m=3$ one always have $s=t$ and the generalized $m$-gon is the flag complex of a projective plane. In that case the smallest positive eigenvalue of the Laplacian is

$$
1-\frac{\sqrt{s}}{s+1}
$$

(3) For $m=4$ the smallest positive eigenvalue of the Laplacian is

$$
1-\sqrt{\frac{s+t}{(s+1)(t+1)}} .
$$

(4) For $m=6$ the smallest positive eigenvalue of the Laplacian is

$$
1-\sqrt{\frac{s+t+\sqrt{s t}}{(s+1)(t+1)}} .
$$

(5) For $m=8$ the smallest positive eigenvalue of the Laplacian is

$$
1-\sqrt{\frac{s+t+\sqrt{2 s t}}{(s+1)(t+1)}} .
$$

4.2. Exotic 2-dimensional affine buildings. Recall 2-dimensional affine building is a 2-dimensional simplicial complex $X$ for which the links of all vertices are generalized thick $m$-gons such that if $(u, v, w)$ is a simplex in $X$ and $X_{u}, X_{v}, X_{w}$ are generalized $m_{u}, m_{v}, m_{w}$-gons respectively, then

$$
\frac{1}{m_{u}}+\frac{1}{m_{v}}+\frac{1}{m_{w}}=1
$$


There is a way to construct affine buildings from local fields, but there are examples of 2-dimensional affine buildings that do not arise from a local field. Below is a table of examples of finite simplicial complexes whose universal cover is an exotic 2-dimensional affine building (i.e. the fundamental group of those complexes acts cocompactly and freely on an exotic building). Those examples are interesting in our context because they fail to meet Żuk's criterion for property (T), but they meet our criterion given in corollary 3.7. In the table below the first column indicates the Coxeter diagram of the finite simplicial complex (with $(s, t)$ written above every link), the second column indicates the universal cover and the last column indicates the reference from which the example was taken.

Table 1. Examples of $\pi_{1}$ groups acting on exotic affine buildings which meet out criterion.

\begin{tabular}{|c|c|c|}
\hline diagram & universal cover & reference \\
\hline$s=t=5 \quad s=t=5$ & $\tilde{G}_{2}$ & [6], $4^{\text {th }}$ example \\
\hline$s=3, t=9 \quad s=9, t=3$ & $\widetilde{C}_{2}$ & [6], $2^{\text {th }}$ example \\
\hline$s=3, t=5 \quad s=3, t=5$ & $\tilde{C}_{2}$ & [7, C.6.10] \\
\hline
\end{tabular}

We will show that the first of those examples meets our criterion (checking the other two examples is left to the reader). In the first example, there are three types of links:

(1) bipartite graph in which $\lambda=1$ and therefore

$$
\bar{\lambda}_{1}=\frac{1}{2}
$$

(2) generalized 3-gon with $s=5$ in which

$$
\bar{\lambda}_{2}=\frac{1}{2}-\frac{\sqrt{5}}{6}
$$

(3) generalized 6-gon with $s=t=5$ in which

$$
\bar{\lambda}_{3}=\frac{1}{2}-\frac{\sqrt{15}}{6} .
$$


Therefore

$$
\bar{\lambda}_{2}+\bar{\lambda}_{3}=1-\frac{\sqrt{5}+\sqrt{15}}{6}<0
$$

so Żuk's criterion does not hold, but

$$
\bar{\lambda}_{1}+\bar{\lambda}_{2}+\bar{\lambda}_{3}=\frac{3}{2}-\frac{\sqrt{5}+\sqrt{15}}{6}>0
$$

and

$$
\begin{gathered}
\left(\bar{\lambda}_{1}+\bar{\lambda}_{2}\right)\left(\bar{\lambda}_{1}+\bar{\lambda}_{3}\right)+\left(\bar{\lambda}_{1}+\bar{\lambda}_{2}\right)\left(\bar{\lambda}_{2}+\bar{\lambda}_{3}\right)+\left(\bar{\lambda}_{1}+\bar{\lambda}_{3}\right)\left(\bar{\lambda}_{2}+\bar{\lambda}_{3}\right)= \\
=3 \frac{5}{9}+\frac{15 \sqrt{3}}{36}-\frac{4}{6}(\sqrt{5}+\sqrt{15})>0
\end{gathered}
$$

therefore the criterion stated in the corollary holds.

Remark 4.1. The first example indicated in the table above is a finite simplicial complex on which the Lyons sporadic group acts. This is the example mentioned in the introduction. As mentioned in the table, more details about this simplicial complex and its connection to the Lyons group can be found in [6] (4 $4^{\text {th }}$ example).

It is also of some interest to indicate which cases property (T) is still unsettled for exotic 2-dimensional affine buildings. The table below compares the minimal $q$ needed to assure property $(\mathrm{T})$ using our Theorem 1 and [10, Theorem 1] for exotic affine buildings of uniform thickness $q$ (i.e. $s=t=q$ for all the generalized polygons). One can see that for buildings of type $\widetilde{C}_{2}$ and $\widetilde{G}_{2}$ there are still unsettled cases when the thickness $q$ is less than 4 .

Table 2. Comparison of criteria for 2 dimensional affine buildings of uniform thickness.

\begin{tabular}{|c|c|c|}
\hline Building type & minimal $q$ by [10, Theorem 1$]$ & minimal $q$ by Theorem 1 \\
\hline$\widetilde{A}_{2}$ & 2 & 2 \\
\hline$\widetilde{C}_{2}$ & 7 & 4 \\
\hline$\widetilde{G}_{2}$ & 7 & 4 \\
\hline
\end{tabular}

4.3. Hyperbolic buildings. A Dynkin diagram is of hyperbolic type, if it is not of finite or affine type, but every proper subdiagram is finite or affine. A Dynkin diagram is of hyperbolic compact type if it is not of finite or affine type, but every proper subdiagram is finite. A Dynkin diagram is called symmetrizable if the associated generalized Cartan matrix can be written as a product of a diagonal matrix and a symmetric matrix. 
For every compact hyperbolic and symmetrizable Dynkin diagram and every finite field $\mathbb{F}_{p^{r}}$, Tits [9] constructed a Kac-Moody group, acting cocompactly on an hyperbolic building with thickness $q=p^{r}+1$. We will deal only with cases in which the Dynkin diagram is symmetrizable, compact that has 3 vertices. Such Dynkin diagram is a triangle with $m_{1}, m_{2}, m_{3}$ labels on the edges where $m_{i}=2,3,4,6$ and $\frac{1}{m_{1}}+\frac{1}{m_{2}}+\frac{1}{m_{3}}<1$. Checking symmetrizablity of the generalized Cartan matrix in this case is straightforward and will not be done here. We note that since our criterion is only depends on the links, we only care about the Coxeter group associate with the diagram and the thickness of the building and not with any finer details of the building structure.

Since the links are again generalized polygons, we can use the results of [5] again for the smallest positive eigenvalue of the Laplacian of each link. The table below compares the minimal $q$ needed to assure property (T) using our Theorem 1 and [10, Theorem 1] (cases in which both theorems give the same $q$ were omitted).

Table 3. Comparison of criteria for 2 dimensional hyperbolic buildings.

\begin{tabular}{|c|c|c|}
\hline Coxeter diagram & minimal $q$ by Theorem 1 & minimal $q$ by [10, Theorem 1] \\
\hline & 10 & 12 \\
\hline
\end{tabular}

\section{References}

[1] P. Abramenko and K. S. Brown, Buildings. Graduate Texts in Mathematics, 248. Springer, New York, 2008. Zbl 1214.20033 MR 2439729

[2] W. Ballmann and J. Światkowski, On $L^{2}$-cohomology and property (T) for automorphism groups of polyhedral cell complexes. Geom. Funct. Anal. 7 (1997), no. 4, 615-645. Zbl 0897.22007 MR 1465598

[3] B. Bekka, P. de la Harpe, and A. Valette, Kazhdan's property (T). New Mathematical Monographs, 11. Cambridge University Press, Cambridge, 2008. Zbl 1146.22009 MR 2415834

[4] J. Dymara and T. Januszkiewicz, New Kazhdan groups. Geom. Dedicata 80 (2000), no. 1-3, 311-317. Zbl 0984.22002 MR 1762517

[5] W. Feit and G. Higman, The nonexistence of certain generalized polygons. J. Algebra 1 (1964), 114-131. Zbl 0170955 MR 0126.05303

[6] W. M. Kantor, Some geometries that are almost buildings. European J. Combin. 2 (1981), no. 3, 239-247. Zbl 0577.00009 MR 0633119 
[7] L. A. Rosati (ed.), Buildings and the geometry of diagrams. (CIME, Como, 1984) Lecture Notes in Mathematics, 1181. Springer, Berlin etc., 1986. Zbl 0514.51006 MR 0843388

[8] Y. Shalom, Rigidity of commensurators and irreducible lattices. Invent. Math. 141 (2000), no. 1, 1-54. Zbl 0978.22010 MR 1767270

[9] J. Tits, Uniqueness and presentation of Kac-Moody groups over fields. J. Algebra 105 (1987), no. 2, 542-573. Zbl 0626.22013 MR 0873684

[10] A. Żuk, La propriété (T) de Kazhdan pour les groupes agissant sur les polyèdres. C. R. Acad. Sci. Paris Sér. I Math. 323 (1996), no. 5, 453-458. Zbl 0858.22007 MR 1408975

Received February 19, 2012

Izhar Oppenheim, Department of Mathematics, Ben Gurion University of the Negev, P.O.B. 653, Be'er Sheva 8410501, Israel

e-mail: izharo@bgu.ac.il 